\title{
Problems of modeling of complex technological systems of greenhouse production
}

\author{
Evgeniya P. Klyuchka, ${ }^{1,}$, Viktor V. Radin ${ }^{1}$, Leonid M. Groshev ${ }^{1}$, and Sergey I. Kambulov ${ }^{2}$ \\ ${ }^{1}$ Don State Technical University, 344000 Rostov-on-Don, Russia \\ ${ }^{2}$ State Scientific Establishment "Agriculture research center "Donskoy", North Caucasus Institute of \\ Mechanization and Electrification of Agriculture, 347740 Zernograd, Russia
}

\begin{abstract}
The main problems connected with modelling of complex technological systems of greenhouse production are considered. The methodological approaches used at present on the basis of classical comparative analysis with subsequent heuristic conclusions are analysed. Based on the study of this issue, it was concluded that the development and implementation of innovative technologies that affect the individual subsystems of the greenhouse complex do not provide the optimal solution to improve the energy efficiency of the system as a whole. There is a need to create a new synergistic technological paradigm for abandoning costly full-scale models and developing models with a high level of adequacy of real technological processes.
\end{abstract}

\section{Introduction}

The complex technological system of greenhouse production is a set of technological and biological objects, technological and information processes, technological and informationcontrol equipment that are in certain relations (connections, interactions). In a complex system of greenhouse production, various processes take place that encompass the metabolism, energy and information. In fact, they consist of three subsystems: biotechnical $\mathrm{S} 1$, technological equipment S2 and information control S3.

The first component S1 is formed by biological objects (plants and people) and a set of engineering equipment included in the general production process, which ensures the operation of all systems of greenhouse production. The second component S2 is formed by technological subsystems with material communication systems (fuel system, electricity and water supply). The totality of technological equipment and material pipelines is a hardware structure. The third component of S3 is represented by information exchange processes and information management equipment (measuring devices, information converters, control mechanisms, etc.). Between the systems S1, S2 and S3 information and resource interactions occur.

Let us consider some properties of a complex technological system of greenhouse production by classifying the processes occurring in them, which will allow us to reveal the essence of the object under study $[1,2,3]$ :

\footnotetext{
* Corresponding author: klyuchkae@mail.ru
} 
- By type of time scale, parallel systems with continuous time (biological processes, weather changes) and systems with discrete time (all technical and control and measuring systems) exist within a single technological process.

- The technological system contains elements with both lumped parameters (equipment) and a distributed (temperature field).

- The structure of the technological system contains both stationary subsystems (normal modes of operation of engineering equipment) and non-stationary ones - the parameters are functions of time (diurnal, seasonal).

- Systems of greenhouse production according to the method of organization of technological processes, depending on the task, can be continuous and periodic actions (successive vegetative stages pass).

This is not a complete list of the properties of the technological system of greenhouse production, but it gives an idea that the complexity of this type of system is determined primarily not by a large number of subsystems and a multilevel structure, but by complexity behavior, a variable set of internal states. Basically, the difficulty lies in the difficulty of detecting (or completely lacking in understanding) certain relationships between subsystems, which makes the individual tasks of the system as a whole insoluble and as a consequence of the difficulties that arise in the management of everything a complex system.

From the analysis of available literature, it turned out that depending on the purpose of the study, modeling objects usually become separate technological processes, technical equipment, measuring instruments, control devices for individual systems, and so on. The most often considered energy is transferred in the form of heat, although there are many other energy processes - optical radiation, mechanical and electrical energy, information flows and others. The simulation of the functioning of a complex technological system of greenhouse production (Fig. 1) for the purpose of optimization has not yet been carried out.

\subsection{The problems of modeling associated with the objects of research}

If we consider the problem of modeling complex technical systems, in which biological objects are included, it is necessary to say about the difference in approaches to this issue of specialists with a purely technical education and specialists with biological knowledge. At present, the problem is absolutely unaffected, but within the framework of this article, for an example, we touch only on two aspects of this problem.

Often a huge mistake in modeling complex technical systems of greenhouse production is the desire to build a model on the concept of photosynthetic productivity. But in fact, photosynthesis is only a separate link, in a large chain of sequentially occurring processes, the productivity of photosynthesis is part of the overall integral result. The competitor to the process of photosynthesis is respiration, and if photosynthesis occurs only in the presence of optical energy, the respiration of plants occurs constantly. Photosynthesis manifests itself as a "rechargeable battery", which periodically becomes infected and discharged. Accumulated energy goes to both breathing and current processes. Consequently, the quality of the product obtained depends entirely on the "characteristics of the charger" of photosynthesis (capacity, volume, etc.). Most likely, the final result of production (by quantity and quality) depends on the process of respiration of plants or on the balance between the processes of photosynthesis and respiration $[4,5,6,7]$. 


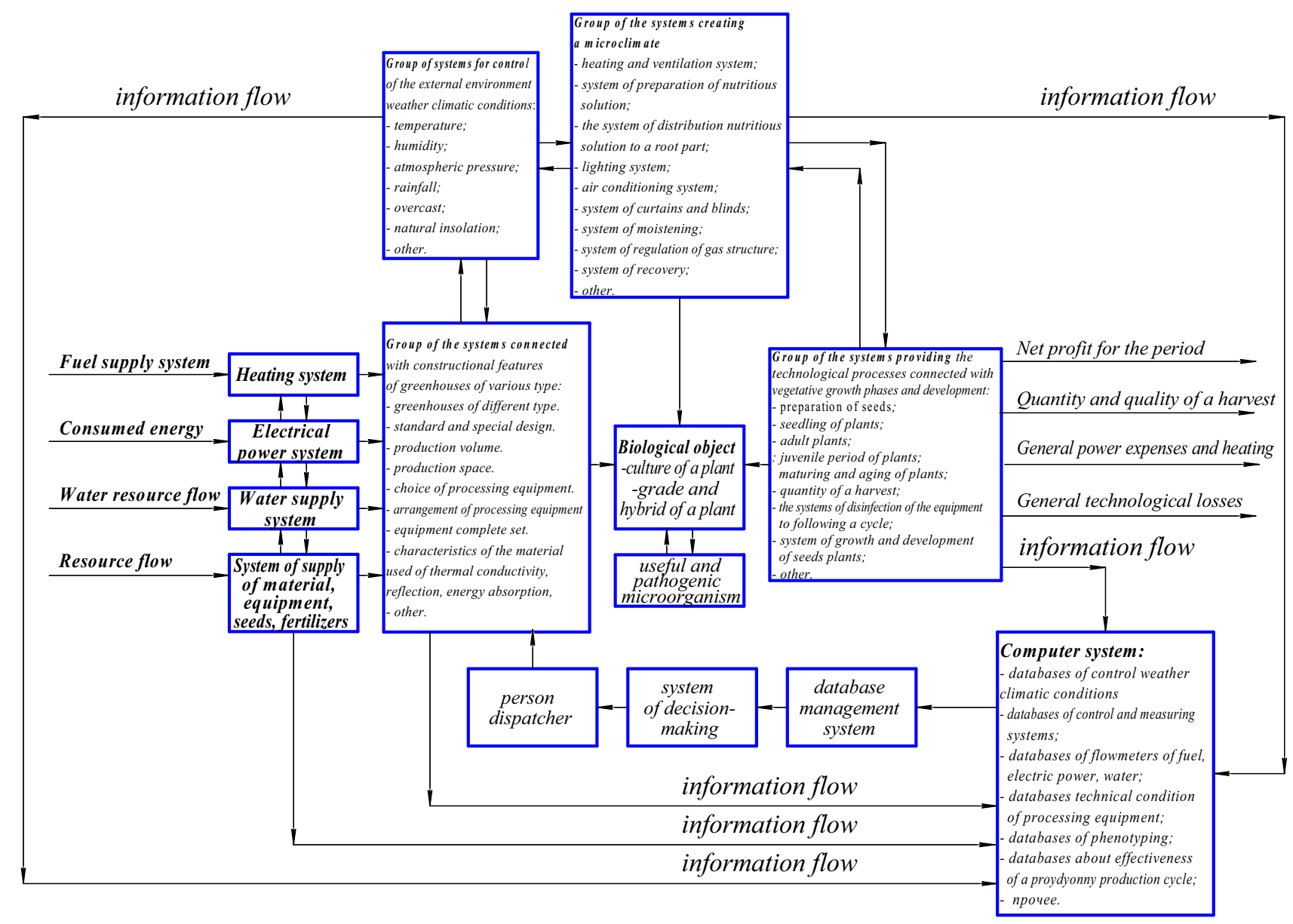

Fig.1. The structural scheme of the subsystems of the technological process of greenhouse production 
This problem is under development. Thus, a rather small knowledge of the properties of the biological object of specialists with technical education introduces an understated negative contribution both in the process of experiment planning and in modeling of greenhouse production systems.

A rather illustrative example, which confirms the foregoing, is the problem of using LED technologies in growing greenhouse plants. The situation has become so exacerbated that there is a need for a new applied field of knowledge about the effects of visible, infrared and ultraviolet radiation on seeds, plants, fruits during their growth, maturation, storage and processing. This science is called "agrophotonics." In the current situation, we observe that LED technologies are at the development stage, with their work there is a huge number of unresolved problems, but this does not prevent the development of a large market for LED products. In the light of this, the most important task of agrophotonics is the collection of evidence base on the effect of optical radiation energy on plants on obtaining a commodity result, as well as the solution of the problem related to metrology, development of measuring instruments and rationing [8].

At present, the flow of information about the effect of LED technology on plants is quite large and saturated. If the experiment is based on the development of a technical solution for LED equipment, this is a rather optimistic conclusion about the use of this technology in greenhouse production. On the contrary, from biologists have a skeptical attitude. In November 2016, the largest business conference in Russia and CIS countries was held on the application of LED technology "10th International Forum of LED Technology LED FORUM 2016" by the organizer, who was Messe Frankfurt RUS, with the support of VNISI him. SI Vavilov. The report was presented at the forum of the Moscow Agricultural Academy (IA Timiryazev). Tarakanova (Doctor of Biological Sciences, Professor, Head of the Department "Plant Physiology") "LED emitters: new opportunities in regulating the growth and development of plants" [9].

The report talked about an experiment with various salad varieties grown under LEDs and under high-pressure gas-discharge sodium lamps. Different varieties of one culture gave mixed results of influence. Earlier, scientists of the Moscow Agricultural Academy (MAAA named after KA Timiryazev) conducted similar studies with strawberry garden (frag aria $\times$ ananassa duch.) Under conditions of light culture [10,11.12].

Analyzing the presented report, it can be noted that the choice of the light source directly depends on both the choice of culture and the varietal characteristics. Thus, the spectral composition of illumination influences biochemical processes and allows to make the internal complex adjustment of the plant at a more subtle level. This circumstance once again emphasizes the individual approach to each plant in the selection of the lighting system, regardless of the chosen culture and varietal characteristics, which significantly complicates the determination of the effectiveness of a particular technical solution of the lighting system.

Usually a plant, as a biological object, is viewed in a somewhat simplified form. In fact, a plant is a single whole organism in which all processes are closely interrelated. The influence of external conditions on any plant is difficult, because in nature all conditions act on the plant simultaneously. And while no one knows where the action of one of them ends and the action of the other begins. It is practically impossible to determine the condition that is decisive in a given period of plant growth and development.

Objective evaluation of the structural diagram of the subsystems of the technological process of greenhouse production in Figure 1, gives an idea of the modern view of this type of production, which is characterized by the lack of "information flow" from the biological object. Now in greenhouse production, it is common practice to assess the state of greenhouse plants on expert opinion from a technologist with agronomic education on a protected ground. A rather contradictory estimate, which depends on the individual 
qualities of a person, on his knowledge base, intuition, experience, and so on. In the Russian greenhouse practice, there is absolutely no connection between the technical systems (subsystems) and the biological object. And, conversely, in foreign practice, there is now an increased interest in phenotyping systems in relation to greenhouse production $[13,14,15]$. Increasing the degree of automation of production to the level of intellectual control systems is to improve the quality and objectivity of management processes, increase yield and product quality, and reduce specific operating costs $[16,17]$.

\section{Conclusion}

Having analyzed the problems existing in modeling a complex technological system of greenhouse production, it should be emphasized that partial modernization of individual systems and subsystems will not solve the fundamental decisive leap from the fourth (in which the Russian greenhouse crop industry is located), bypassing the fifth - in the sixth technological order units) $[18,19,20]$. For this, it is necessary to pay attention to the solution of important conceptual directions:

- The adequacy of the true measurement and reporting of multiple parameters in the science of plants is an undeniable need to ensure that research around the world can be compared, interpreted, reproduced in different laboratories. This is one of the conditions for the development of applied science Agrophotonics.

- Greenhouse production should have control and measurement systems based on the principle of phenotyping and on the principles of bioinformatics. This is a necessary condition for establishing the connection of a biological object with engineering and technical systems, united by a single technological process.

- Improvement of information systems, automation systems, to the full robotization of all greenhouse production.

- Hothouse production must have closed systems containing recycles.

On the basis of the study, the conclusion is drawn that the development and implementation of innovative technologies affecting individual subsystems of greenhouse production (aerohydroponics, LED technologies, lighting systems and methods, stimulation of plants by fields of various nature, etc.) will not solve the problem of reducing the energy intensity of existing complex technological systems in whole. There is a need for a new synergistic (unifying, complementary, reinforcing) technological paradigm, which will allow creating high-level models of adequacy to real processes and designs instead of costly in-situ models, which will significantly reduce energy and production costs.

\section{References}

1. I.A. Gerasimova, Epistemology and philosophy of science, II (2) 123 (2012)

2. Yu.A. Dubov, S.I. Travkin, V.N. Yakimets, Multicriteria models for the formation and selection of system variants (Nauka, Moscow, 1986)

3. Yu.H. Vermishev, Methods of automatic solutions search for the design of complex technical systems (Radio and Communication, Moscow, 1982)

4. V.I. Chikov, Soros Educational Journal, 12, 23 (1997)

5. V.I. Chikov, G.G. Bakirova, Plant Physiology, 51, 3, 466 (2004)

6. V.I. Chikov, Plant Physiology, 55, 1, 140 (2008)

7. V.I. Chikov, Agricultural Biology, 1, 72 (2012)

8. www.ledforum-moscow.ru 11th International Forum on LED Technologies 7-8 November 2017 Expocentre Fairgrounds, Moscow (Circulation date 05/08/2018) 
9. http://www.lightingmedia.ru Conference «Modern light engineering and energy saving technologies of agrophotonics», Kazan (2018) (Circulation date 05/08/2018)

10. M.N. Yakovtseva, G.F. Govorova, I.G. Tarakanov, Izvestiya Timiryazev Agricultural Academy, 3, 25 (2015)

11. M.N. Yakovtseva, G.F. Govorova, I.A. Bulanova, I.G. Tarakanov, Izvestiya T.KhA, 4, 69 (2016)

12. M.N. Yakovtseva, Photomorphogenetic regulation of growth and development of strawberry garden (Fragaria $\times$ ananassa L.) in conditions of light culture. Dissertation for the degree of candidate of biological sciences on specialty 03.01.05 (MAAA them. K.A. Timiryazev, Moscow, 2017)

13. G.N. Tiwari, Greenhouse technology for controlled environment (Alpha Science International Ltd., UK, 2003)

14. H. Poorter, F. Foriani, M. Stitt, U. Schurr, A. Finck, Y. Gibon et al, Funct Plant Biol, 38,821 (2012)

15. ANSI/ASABE EP411.5 Guidelines for measuring and reporting environmental parameters for plant experiments in growth chambers: American Society for Agricultural and Biological Engineers, USA (2012)

16. I.Yu. Chazova, Milk farm, 1 (25), 187 (2017)

17. I.Yu. Chazova, Organizational and economic mechanism of sustainable development of the vegetable market for protected soil. Thesis for a doctoral degree in economics by specialty 08.00.05 (Izhevsk, 2017)

18. I.A. Gerasimova, Irreducibility of uncertainty in social assessment of technology / Epistemology and philosophy of science, II, 2, 123 (2012)

19. E.V. Pilipenko, O.I. Pechonik, Actual problems of the humanities and natural sciences, 10-1, 225 (2013)

20. M.A. Parshin, D.A. Kruglov, «Modern scientific research and innovation», 5 (37) (2014) Circulation date 05/08/2018 http://web.snauka.ru/issues/2014/05/33059 